\title{
Detection and imaging of quorum sensing in Pseudomonas aeruginosa biofilm communities by surface-enhanced resonance Raman scattering
}

\author{
Gustavo Bodelón ${ }^{1 \star}$, Verónica Montes-García ${ }^{1}$, Vanesa López-Puente ${ }^{1}$, Eric H. Hill², Cyrille Hamon², \\ Marta N. Sanz-Ortiz ${ }^{2}$, Sergio Rodal-Cedeira ${ }^{1}$, Celina Costas $^{1}$, Sirin Celiksoy ${ }^{1}$, Ignacio Pérez-Juste ${ }^{1}$, \\ Leonardo Scarabelli ${ }^{2}$, Andrea La Porta ${ }^{2}$, Jorge Pérez-Juste ${ }^{1}$, Isabel Pastoriza-Santos ${ }^{1}$ \\ and Luis M. Liz-Marzán ${ }^{1,2,3,4 \star}$
}

\begin{abstract}
Most bacteria in nature exist as biofilms, which support intercellular signalling processes such as quorum sensing (QS), a cellto-cell communication mechanism that allows bacteria to monitor and respond to cell density and changes in the environment. As QS and biofilms are involved in the ability of bacteria to cause disease, there is a need for the development of methods for the non-invasive analysis of QS in natural bacterial populations. Here, by using surface-enhanced resonance Raman scattering spectroscopy, we report rationally designed nanostructured plasmonic substrates for the in situ, label-free detection of a QS signalling metabolite in growing Pseudomonas aeruginosa biofilms and microcolonies. The in situ, non-invasive plasmonic imaging of QS in biofilms provides a powerful analytical approach for studying intercellular communication on the basis of secreted molecules as signals.
\end{abstract}

$\longrightarrow$ uorum sensing (QS) is a cell-to-cell communication phenomenon used by bacteria to monitor their local environment and population density by producing, secreting and sensing small diffusible signals called autoinducers, resulting in the synchronous regulation of gene expression on a populationwide $s c^{1,2}$. In numerous bacterial genera, QS-regulated genes have been linked to a wide range of physiological processes such as cell differentiation, antibiotic production, stress tolerance, virulence or biofilm formation, all of which are crucial for survival and pathogenicity ${ }^{3}$. Thus, understanding and controlling this chemical communication system could lead to medical and industrial applications ${ }^{4}$. The elucidation of QS systems is based on planktonic (free-swimming) bacteria grown in shaken liquid cultures, given that bacteria are physiologically similar under these growth conditions. However, in natural environments most bacterial populations exist as densely packed multicellular aggregates embedded in self-produced extracellular polymeric substances often referred to as biofilms. Bacterial biofilms represent a protected mode of growth that has been shown to profoundly affect human health and industrial productivity ${ }^{5}$. In this environment, biofilm bacteria are distinct from their planktonic counterparts in terms of genetic program and physiology ${ }^{6}$. Remarkably, although the high cell density within biofilms provides an optimal environment for intercellular signalling processes including QS, chemical communication in this habitat is also strongly influenced by numerous biological, chemical and physical parameters ${ }^{3,7,8}$.

Pseudomonas aeruginosa is an opportunistic human pathogen responsible for acute and chronic infections, particularly in individuals with compromised immune systems and host defences.
Several lines of evidence indicate that the persistence and antibiotic resistance of this bacterium are due to its inherent capacity to form biofilms, which are often found in the lungs of cystic fibrosis patients, chronic wounds or in medical foreign bodies or indwelling devices ${ }^{9}$. The QS network of $P$. aeruginosa is known to regulate the expression of key virulent factors that can contribute to the bacterial pathological process ${ }^{10}$. Nevertheless, QS-deficient clinical isolates occur and are still capable of causing infections in humans ${ }^{11}$. Due to the links between QS, biofilms and virulence, together with its clinical relevance, $P$. aeruginosa has become a model system to study these two important aspects of bacterial social behaviour. However, despite much research, fundamental questions still remain unanswered regarding the role and mechanisms of QS signalling processes in the context of biofilms $s^{8,12}$, as well as their relevance in human infections ${ }^{11}$.

Traditionally, optical monitoring of QS dynamics employs bacterial biosensors bearing heterologous reporter genes that express bioluminescent or fluorescent proteins in response to QS signalling molecules ${ }^{13,14}$. Although this approach has greatly expanded our understanding of QS, it involves the use of genetically modified bacteria. Thus, the development of alternative methods to detect and image QS-regulated processes with no need for genetic manipulation or labelling is highly desirable towards understanding this form of bacterial communication in natural populations.

Surface-enhanced Raman scattering (SERS) spectroscopy is an ultrasensitive analytical technique ${ }^{15-17}$ that can be applied noninvasively for label-free detection and imaging of a wide range of molecules ${ }^{18-20}$. SERS allows identification of the specific spectral fingerprint of a probe molecule in contact with a plasmonic

${ }^{1}$ Departamento de Química Física, Universidade de Vigo, 36310 Vigo, Spain. ²Bionanoplasmonics Laboratory, CIC biomaGUNE, Paseo de Miramón 182 , 20009 Donostia-San Sebastián, Spain. ${ }^{3}$ Ikerbasque, Basque Foundation for Science, 48013 Bilbao, Spain. ${ }^{4}$ Biomedical Research Networking Center in Bioengineering, Biomaterials, and Nanomedicine (CIBER-BBN), 20009 Donostia - San Sebastián, Spain. *e-mail: gbodelon@uvigo.es;

llizmarzan@cicbiomagune.es 
a

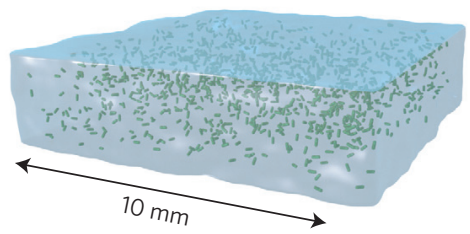

c

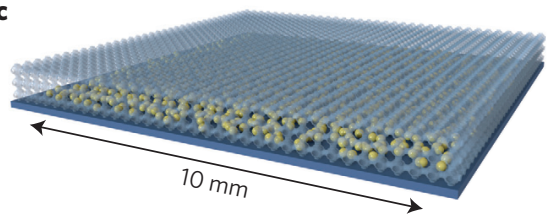

e

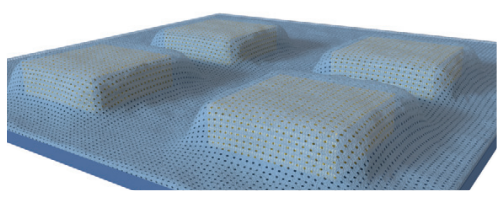

b

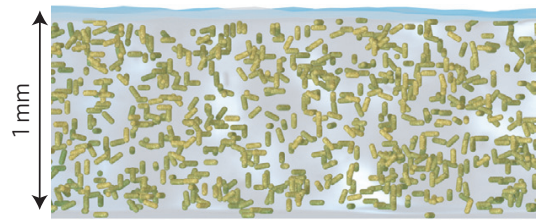

d

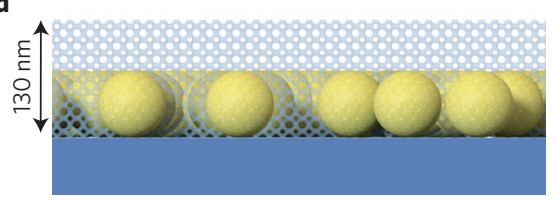

f

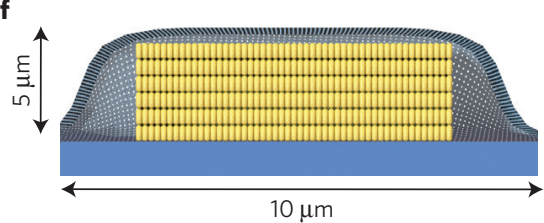

Figure 1 | Schematic representation of nanostructured porous substrates for in situ plasmonic detection and imaging of QS in Pseudomonas aeruginosa

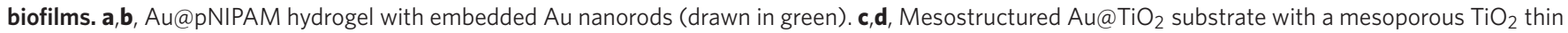
film over a submonolayer of Au nanospheres. e,f, Micropatterned Au@SiO 2 supercrystal array comprising Au nanorods organized in micrometre-sized pedestal-like structures coated with mesoporous silica. Panels b,d,f represent cross-sectional views.

nanostructure and its sensitivity can go as far as the single-molecule level ${ }^{21}$, in particular when the frequency of the excitation laser is in resonance with an electronic transition of the molecule, which is known as surface-enhanced resonance Raman scattering (SERRS). Importantly, SERS/SERRS offers unambiguous identification of analytes, multiplexing capability, requires little or no sample preparation and provides high spatial resolution. The implementation of plasmonic nanoparticles as SERS sensors has set the basis for numerous high-performance in vitro analytical bioassays ${ }^{22,23}$. However, due to inherent limitations of SERS ${ }^{19}$, the direct detection of target analytes in complex biological environments using plasmonics is still in its infancy. One of these limitations is related to the required access to the metal surface in the plasmonic substrate of the relevant analyte, while avoiding signal contamination by other biomolecules, such as proteins, lipids and so on.

We focus this work on pyocyanin, a heterocyclic nitrogencontaining compound of the phenazine family produced by $P$. aeruginosa, which is excreted into the environment where it displays a wide variety of biological activities ${ }^{24,25}$. This phenazine acts as an antibiotic and as a virulence factor in infected hosts, which is in general due to its capacity to generate reactive oxygen species. Remarkably, pyocyanin functions as an intercellular signalling molecule in the QS network of $P$. aeruginos $a^{26}$ and plays important roles in biofilm morphogenesis in this organism ${ }^{10,27-29}$. The biosynthesis of pyocyanin is regulated by the interdependent QS circuits of $P$. aeruginosa in response to environmental factors including cell density ${ }^{10,30,31}$. Since pyocyanin expression is controlled by QS and the possibility of SERS detection has been reported ${ }^{32}$, we implemented a plasmonic approach towards in situ SERRS detection and imaging of pyocyanin, as a proxy of QS, in biofilms and microcolonies of $P$. aeruginosa. Our strategy involves the use of hybrid materials comprising a plasmonic component within a porous matrix that allows diffusion of only small molecules ${ }^{33}$. With this concept in mind and aiming at providing different analytical tools to investigate this form of bacterial communication in live bacterial sessile communities, we fabricated three types of cell-compatible plasmonic platform (Fig. 1). Macroporous poly- $N$-isopropylacrylamide (pNIPAM) hydrogels loaded with Au nanorods (Au@pNIPAM), devised as a highly porous platform with enhanced diffusivity, led to plasmonic detection of pyocyanin homogeneously in both colonized and non-colonized regions of the substrate. On the other hand, mesostructured $\mathrm{Au} @ \mathrm{TiO}_{2}$ substrates bearing a mesoporous $\mathrm{TiO}_{2}$ thin film over a submonolayer of $\mathrm{Au}$ nanospheres restricted pyocyanin detection to biofilm-colonized surfaces with a spatial resolution of about $20 \mu \mathrm{m}$. This platform allowed us to generate SERRS maps to reveal variation of QS plasmonic signal in bacterial biofilms up to millimetre-scale areas. Finally, mesoporous silica-coated micropatterned supercrystal arrays of $\mathrm{Au}$ nanorods $\left(\mathrm{Au} @ \mathrm{SiO}_{2}\right)$, with an extremely high electromagnetic enhancement factor, enabled plasmonic detection of QS behaviour (for example, pyocyanin expression) at early stages of biofilm formation and allowed imaging of the phenazine produced by small clusters of bacteria colonizing micrometre-sized plasmonic features $\left(25 \mu \mathrm{m}^{2}\right.$ on average). Details on substrate fabrication, characterization and analysis of the SERS performance with model analytes are provided in the Methods and Supplementary Sections 1 and 2. This approach, combining purpose-designed nanostructured hybrid materials and SERS, provides an efficient and versatile tool for label-free molecular detection of QS communication in live microbial communities and may thus contribute to understanding biofilm sociomicrobiology and other cellular processes mediated by small, SERS-active biomolecules.

\section{Raman and SERRS characterization of pyocyanin}

Pyocyanin exhibits a broad absorption band in the visible (550-900 nm, Fig. 2a), which has been ascribed to a HOMO-LUMO transition (Supplementary Section 3). Illumination of an aqueous solution of commercial pyocyanin with either $633 \mathrm{~nm}$ or $785 \mathrm{~nm}$ laser lines leads to resonance Raman (Fig. 2b; see a detailed description of the optical properties and vibrational assignment in Supplementary Section 3). This band is not present in any of the other phenazines that may be produced in the $P$. aeruginosa culture (see Supplementary Section 3). Therefore, SERRS, rather than SERS, spectra of commercial pyocyanin were recorded in the wet state after immersion of the plasmonic composites in aqueous pyocyanin solutions of known concentration (between $10 \mathrm{fM}$ and $10 \mu \mathrm{M}$, with tenfold increments) and processed using the SERS spectrum of the plasmonic composite material as a blank. On equilibration, the SERRS spectrum of pyocyanin is clearly visible 

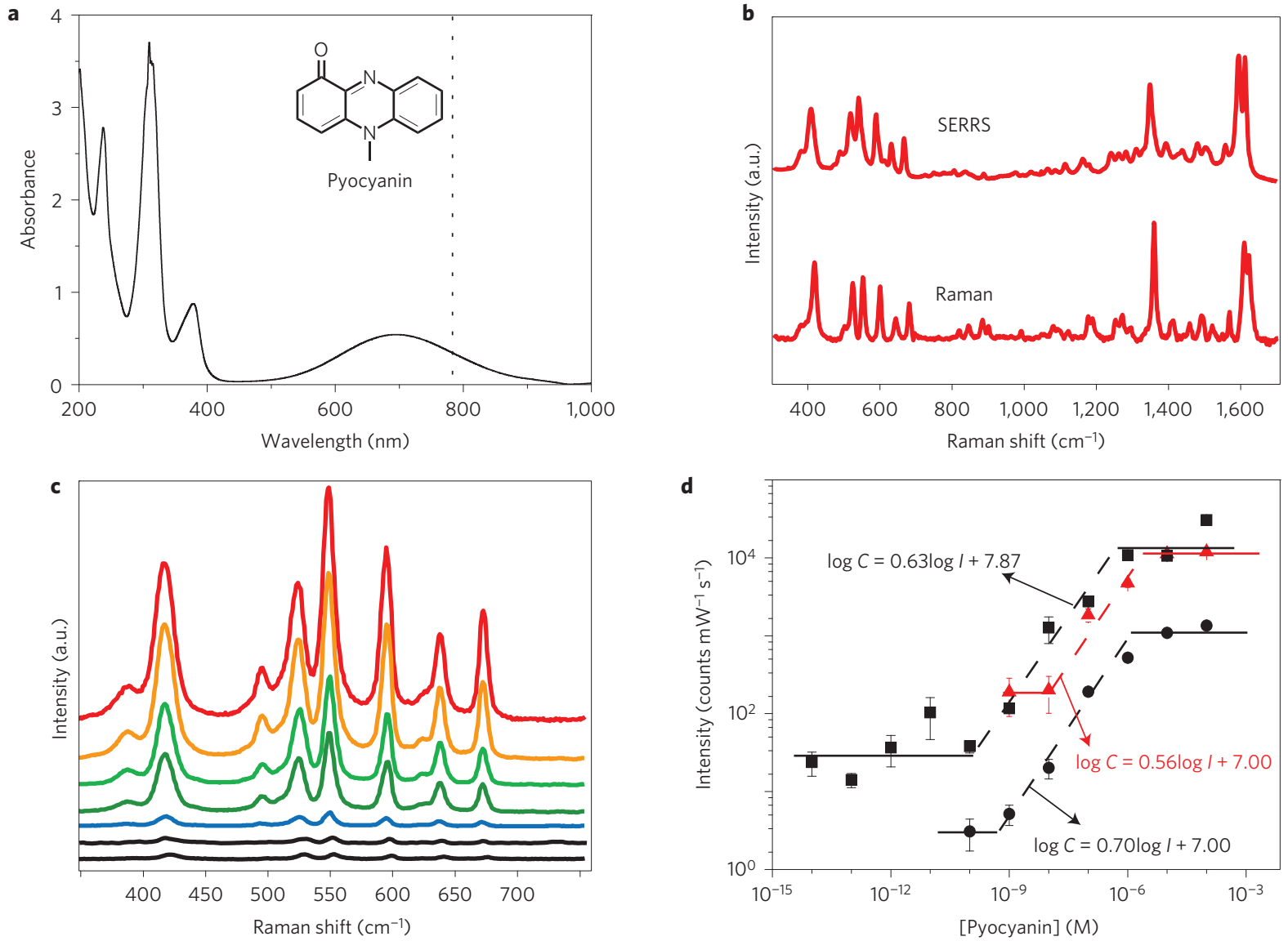

Figure 2 | Raman and SERRS spectra of pyocyanin. a, UV-visible-near-infrared spectrum of aqueous pyocyanin solution (10 $\left.{ }^{-4} \mathrm{M}\right)$ and molecular structure of pyocyanin (inset). The dotted line indicates $785 \mathrm{~nm}$, corresponding to the excitation wavelength used for Raman and SERRS spectra. $\mathbf{b}$, Resonance Raman and SERRS spectra of pyocyanin measured in solid state and in aqueous solution (1 $\mu$ M, Au@pNIPAM hydrogel), respectively. Raman measurement was carried out with a $50 \times$ objective, a maximum power of $54.22 \mathrm{~kW} \mathrm{~cm}^{-2}$ and an acquisition time of $10 \mathrm{~s}$. SERRS measurement was carried out with a $20 \times$ objective, a maximum power of $4.24 \mathrm{~kW} \mathrm{~cm}^{-2}$ and an acquisition time of $10 \mathrm{~s}$. c, Pyocyanin SERRS spectra at different concentrations (between $0.1 \mathrm{nM}$ and $100 \mu \mathrm{M}$ with tenfold-increase steps), recorded on Au@pNIPAM hydrogels. SERRS measurements were carried out with a 20x objective, a maximum power between $0.26-4.24 \mathrm{~kW} \mathrm{~cm}^{-2}$ and an acquisition time of $10 \mathrm{~s} . \mathbf{d}, \mathrm{SERRS}$ intensity (at $418 \mathrm{~cm}^{-1}$ for Au@pNIPAM (circles) and Au@TiO 2 (triangles); at 1,600 $\mathrm{cm}^{-1}$ for micropatterned $\mathrm{Au} @ \mathrm{SiO}_{2}$ (squares)) as a function of pyocyanin concentration. The dashed lines are linear fits in the quantitative detection regions. Each data point corresponds to the average signal from five spectra collected from three different substrates. An excitation laser line at $785 \mathrm{~nm}$ was used for all measurements. Error bars indicate standard deviation. A $20 \times$ objective, a maximum power of $0.98 \mathrm{~kW} \mathrm{~cm}^{-2}$ and an acquisition time of $0.1 \mathrm{~s}$ was used for micropatterned $\mathrm{Au} @ \mathrm{SiO}_{2}$ substrate.

(Fig. 2b). It was noted that for the three selected plasmonic platforms the SERRS signal was found to have low variability from substrate to substrate. Quantitative SERRS detection can be achieved when changes in SERRS signal correspond to changes in analyte concentration in a predictable manner. Therefore, the quantitative detection range was evaluated for all three plasmonic substrates by measuring the SERRS spectra of pyocyanin solutions from $0.1 \mathrm{nM}$ to $0.01 \mathrm{mM}$ with tenfold-increase steps. Figure $2 \mathrm{c}$ shows the SERRS spectra of pyocyanin at different concentrations measured from the Au@pNIPAM substrate with the $785 \mathrm{~nm}$ laser line (similar spectra were obtained for the other substrates). The SERRS measurements showed that quantitative detection of pyocyanin can be achieved in a concentration range that depends on the plasmonic substrate: from $0.1 \mu \mathrm{M}$ down to $10 \mathrm{nM}$ for mesostructured $\mathrm{Au} @ \mathrm{TiO}_{2}$ and Au@pNIPAM hydrogel; from $0.1 \mu \mathrm{M}$ down to $1 \mathrm{nM}$ for the micropatterned mesoporous $\mathrm{Au@SiO}$ 2 substrate (Fig. 2d). Within these concentration ranges, pyocyanin concentration can be expressed quantitatively by empirical formulae: $\log C=0.70 \times$ $\log I+7.00\left(R^{2}=0.97\right)$ for Au@pNIPAM hydrogel, $\log C=0.56 \times$ $\log I+7.00\left(R^{2}=0.91\right)$ for $\mathrm{Au} @ \mathrm{TiO}_{2}$ substrate, and $\log C=0.63 \times$ $\log I+8.68\left(R^{2}=0.97\right)$ for $\mathrm{Au} @ \mathrm{SiO}_{2}$ substrate, where $C$ is the pyocyanin concentration (in molar concentration) and $I$ is the SERRS intensity (in kilo-counts $\mathrm{mW}^{-1} \mathrm{~s}^{-1}$ ) (ref. 34). Above each concentration range the SERRS signal intensity will be saturated. Interestingly, for the micropatterned $\mathrm{Au} @ \mathrm{SiO}_{2}$ supercrystal array, when the concentration is lower than $0.1 \mathrm{nM}$ the signal is not homogeneous across all pillars and detection of molecular binding events required SERRS mapping through several pillars. This led to ultrasensitive detection of pyocyanin down to a limit of detection (LOD) of $10^{-14} \mathrm{M}$ (Fig. 2d), in agreement with the reported plasmonic properties for this type of nanostructure ${ }^{35}$.

\section{SERRS analysis of phenazines in planktonic cultures}

The high performance of our plasmonic substrates allowed us to focus on the detection of pyocyanin produced in vitro by $P$. aeruginosa PA14 bacteria. We first investigated pyocyanin expression in planktonic cultures grown under constant agitation, using Au@pNIPAM hydrogels as plasmonic substrates. To this end, bacteria-free samples obtained from late stationary-phase cultures grown for $20 \mathrm{~h}$ were incubated for several hours with the plasmonic substrates before SERRS measurements. As shown in Fig. 3a, the sample obtained from wild-type $P$. aeruginosa PA14 bacteria 

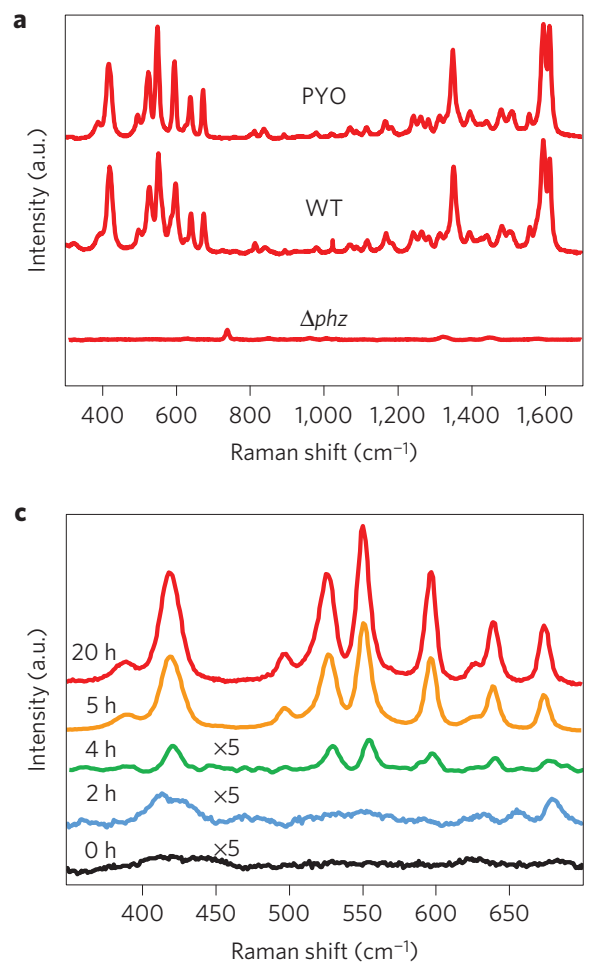
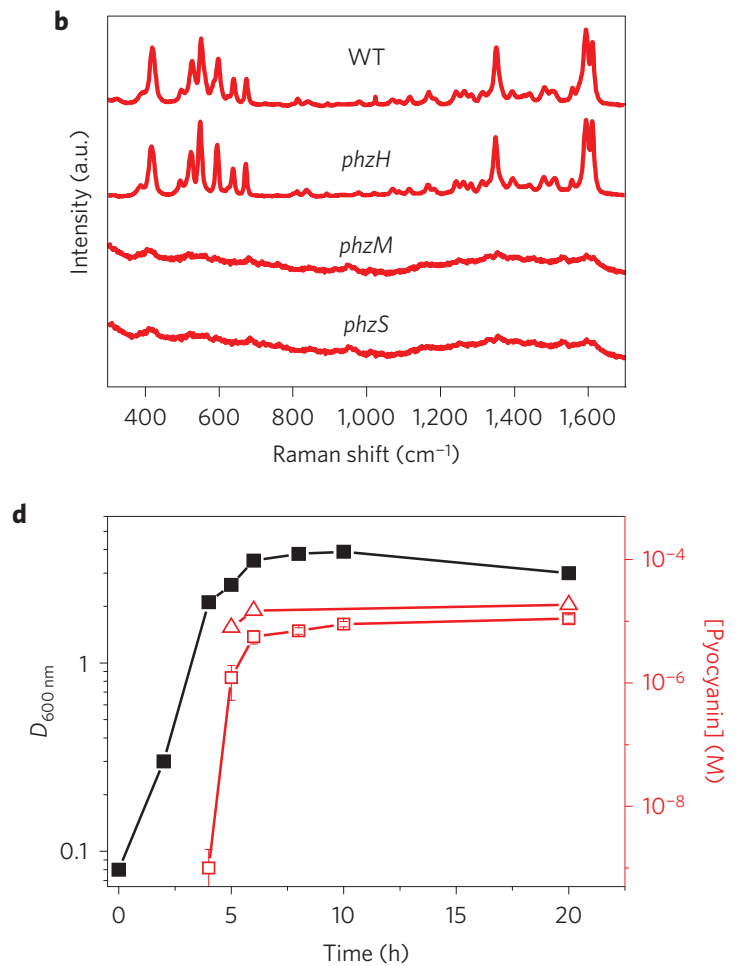

Figure 3 | SERRS analysis of phenazine production by P. aeruginosa PA14 strains grown in planktonic cultures, recorded with Au@pNIPAM hydrogels. a, SERRS spectra of commercial pyocyanin (PYO) and of pyocyanin produced by the wild-type (WT) and the phenazine-null $\Delta$ phz1/2 ( $\Delta$ phz) strains. b, SERRS spectra of pyocyanin produced by the wild-type and the indicated phz mutant strains. c, SERRS analysis of pyocyanin release by the $P$. aeruginosa PA14 strain at different growth times. For clarity, the spectra noted with $\times 5$ have been multiplied by a factor of 5 . d, Growth curve of $P$. aeruginosa PA14 by measuring attenuance of the liquid culture at $600 \mathrm{~nm}$ (black squares) and pyocyanin concentration determined by SERRS (red squares) and UV-visible spectroscopy (red triangles). All SERRS measurements were performed with a $785 \mathrm{~nm}$ laser line employing a $20 \times$ objective, maximum power between $1.72 \mathrm{~kW} \mathrm{~cm}^{-2}$ and an acquisition time of $10 \mathrm{~s}$ (intensity at $418 \mathrm{~cm}^{-1}$ ). The errors bars represent the standard deviation of three different measurements.

displayed a SERRS fingerprint almost identical to that of commercial pyocyanin, whereas no pyocyanin signal was detected in a sample from a phenazine-null mutant strain $(\Delta p h z 1 / 2)$. P. aeruginosa PA14 produces at least five types of phenazines, a precursor termed phenazine-1-carboxylic acid (PCA) that can be converted into 1-hydroxyphenazine (1-HO-PHZ), phenazine-1-carboxamide (PCN), 5-methylphenazine-1-carboxylic acid betaine (5-MCA) and pyocyanin, by action of the enzymes $\mathrm{PhzS}$, PhzH and PhzM (see Supplementary Fig. 4.1 for details) ${ }^{10}$. To confirm that the SERRS signals specifically correspond to pyocyanin and not to any of the other four phenazines, cell-free samples obtained from planktonic cultures of different $P$. aeruginosa PA14 mutants were analysed. The results (Fig. 3b) confirm that only the phzH mutant yielded the characteristic SERRS fingerprints of pyocyanin, meaning that the spectrum is indeed pyocyanin-specific, which is likely to be related to the additional enhancement under resonant Raman conditions.

Next, the release of pyocyanin into the extracellular medium throughout the growth of planktonic wild-type $P$. aeruginosa PA14 was investigated. Aliquots from the culture were collected at various times and used for measurement of the concentration of cells through attenuance at $600 \mathrm{~nm}\left(D_{600 \mathrm{~nm}}\right)$, enumeration of viable bacteria and determination of pyocyanin concentration by SERRS and UV-visible spectroscopy at $691 \mathrm{~nm}\left(\lambda_{\max } \text { of pyocyanin }\right)^{36}$ following chloroform extraction (Supplementary Section 4). Extraction with chloroform is a common strategy for pyocyanin purification, which allowed us to interpolate SERRS intensities to the calibration curve and determine the corresponding concentrations. Representative sequential SERRS spectra shown in Fig. $3 \mathrm{c}$ demonstrate the presence of pyocyanin already at four hours after the start of a culture bearing a $D_{600 \mathrm{~nm}}$ of 2.1 (Fig. $3 \mathrm{~d}$ ) and $2.9 \times 10^{9}$ c.f.u. $\mathrm{ml}^{-1}$. Interpolation of the SERRS signal to the calibration curve yielded a concentration of pyocyanin of approximately $1 \mathrm{nM}$ (Fig. 3d). Interestingly, just one hour later, on reaching an attenuance of 2.6 and $3.2 \times 10^{9}$ c.f.u. $\mathrm{ml}^{-1}$, the SERRS signal increased significantly (Fig. $3 c$ ), indicating a concentration of $1 \mu \mathrm{M}$ (Fig. 3d). This abrupt increase in pyocyanin concentration parallels the well-known exponential growth of bacterial cultures. At later times, the SERRS intensities and the amount of pyocyanin did not increase significantly and remained constant within the micromolar concentration range (Fig. $3 \mathrm{c}, \mathrm{d}$ ) as previously reported by UV-visible spectroscopy ${ }^{26}$. Indeed, the amount of pyocyanin measured by SERRS correlated to parallel measurements by UV-visible spectroscopy (Fig. 3d). As expected, better sensitivity was achieved by SERRS, enabling the detection of pyocyanin in bacterial cultures at subnanomolar concentration, whereas UVvisible spectroscopy was not reliable below micromolar concentration levels ${ }^{26}$ (Fig. 3d). These results are also in agreement with those of ref. 32, which reported the use of a silver nanorod substrate for SERS quantification of pyocyanin (LOD of $2.38 \times 10^{-8} \mathrm{M}$ ) extracted from planktonic cultures of a P. aeruginosa PAO1 strain ${ }^{32}$. Mesostructured Au@ $\mathrm{TiO}_{2}$ and micropatterned Au@SiO ${ }_{2}$ substrates also allowed us to detect pyocyanin purified from planktonic cultures. Notably, whereas $\mathrm{Au} @ \mathrm{TiO}_{2}$ showed a similar sensitivity to that of Au@pNIPAM, Au@SiO $\mathrm{Sip}_{2}$ supercrystals yielded pyocyanin detection from a planktonic culture having $3.4 \times 10^{7}$ c.f.u. $\mathrm{ml}^{-1}$, two orders of magnitude less than that required when the Au@pNIPAM hydrogel was used.

In situ detection of quorum sensing in biofilm communities Once we confirmed the suitability of the proposed substrates towards plasmonic detection of pyocyanin, we explored their application as platforms for sustaining bacterial growth and in situ SERRS detection of the phenazine in developing biofilms. At the 

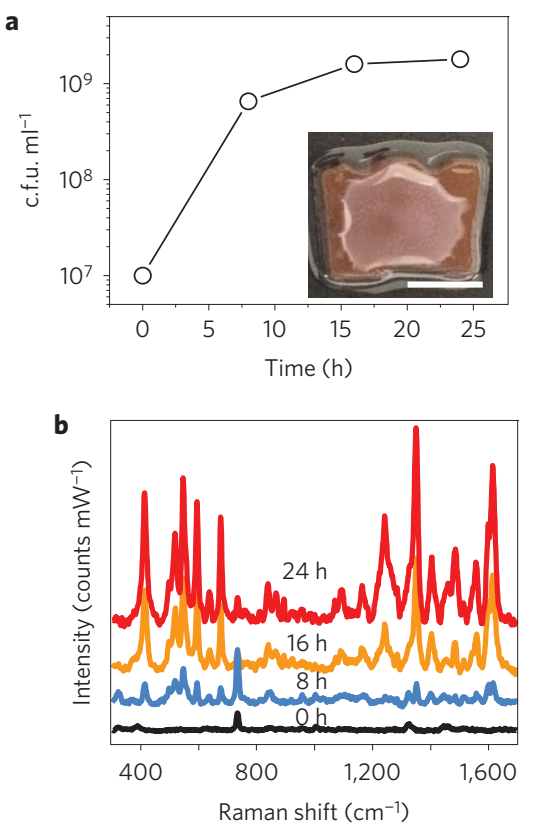

c

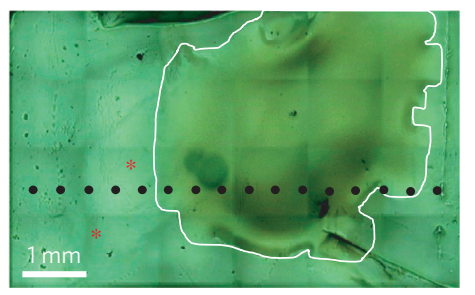

d

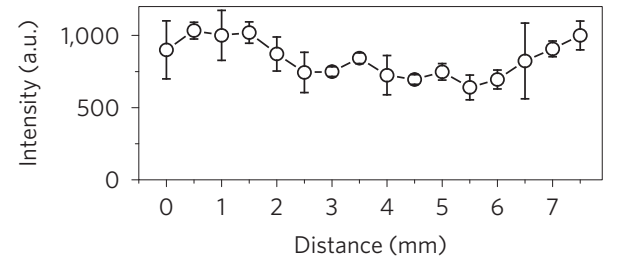

e

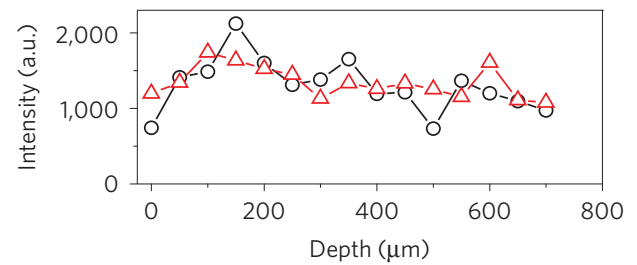

f
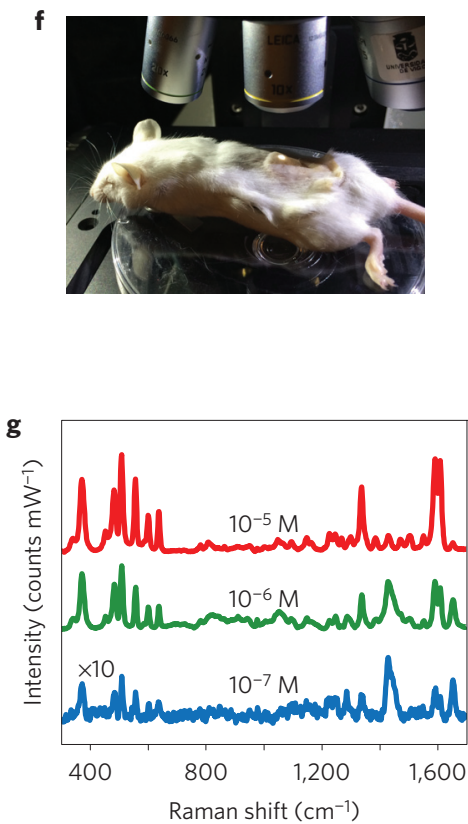

Figure 4 | In situ detection of pyocyanin secreted by P. aeruginosa PA14 colony-biofilms grown on Au@pNIPAM hydrogels. a, Graphical representation of viable bacteria (c.f.u. $\mathrm{ml}^{-1}$ ) quantified over time. The inset shows an image of the colony-biofilm (scale bar, $0.5 \mathrm{~cm}$ ). $\mathbf{b}$, SERRS spectra recorded at the indicated times. c, Optical micrograph of a bacterial colony-biofilm, which is outlined in white for clarity. $\mathbf{d}$, SERRS intensities $\left(418 \mathrm{~cm}^{-1}\right) \mathrm{measured}$ in $\mathbf{c}$ at the places indicated with black dots and plotted as a function of distance. The error bars show the standard deviation from three different measurements. e, SERRS intensities $\left(418 \mathrm{~cm}^{-1}\right)$ measured in $\mathbf{c}$ at the places indicated with asterisks and plotted as a function of the depth from the surface. SERRS measurements of colony-biofilms were done using a $785 \mathrm{~nm}$ laser line for $10 \mathrm{~s}$ and using a maximum power of $0.91 \mathrm{~kW} \mathrm{~cm}^{-2}$ employing a $20 \times$ objective. f, Photograph showing the Raman experimental set-up for detection of pyocyanin in subcutaneous implants in mice. g, Under-skin SERRS spectra of pyocyanin spiked at the indicated concentrations on Au@pNIPAM hydrogel. SERRS measurements of pyocyanin-spiked hydrogels were performed using a $785 \mathrm{~nm}$ laser line for $10 \mathrm{~s}$ using a maximum power of $24.45 \mathrm{~kW} \mathrm{~cm}^{-2}$ employing a $10 \times$ objective. For clarity, the spectra noted with $\times 10$ have been multiplied by a factor of 10 .

initial phase of biofilm formation, planktonic bacteria attach to biotic or abiotic surfaces within minutes, followed by aggregation into microcolonies, a step that is sometimes described as the first social trait in the development of a biofilm. Such bacterial aggregates support the production and accumulation of extracellular polymeric substances that serve as scaffolds for the bacterial community and aid in the formation of a mature biofilm 5 . Numerous approaches have been developed for the study of these sessile microbial communities ${ }^{37,38}$. The so-called 'colony-biofilm assay' refers to a model for in vitro study of bacterial biofilms grown over an agarbased medium under static growth (that is, with no agitation). This model has been previously used for studying the role of phenazines in biofilm morphogenesis ${ }^{27}$, as well as for the electrochemical characterization of QS in P. aeruginosa biofilms ${ }^{39,40}$.

We adapted the colony-biofilm assay to assess the suitability of Au@pNIPAM hydrogels as platforms for sustaining bacterial growth and in situ SERRS detection of pyocyanin. Dry plasmonic hydrogels were infused with liquid nutrients by swelling in Luria-Bertani (LB) medium and then inoculated with a bacterial suspension of $P$. aeruginosa PA14 previously depleted of extracellular pyocyanin (Supplementary Section 4). As shown in Fig. 4, the substrate indeed allowed bacterial proliferation as a colony on its surface, as well as plasmonic detection of pyocyanin secretion into the underlying sensor. SERRS measurements recorded from different spots over the substrate surface, both inside and outside the region colonized by the colony-biofilm (Fig. 4c), revealed horizontal pyocyanin diffusion towards regions located as far as $2 \mathrm{~mm}$ away from the biofilm margin (Fig. 4d). Interestingly, the intensity of the SERRS signals was slightly higher in non-colonized regions, most likely due to interferences caused by the biofilm matrix. Vertical diffusion was also evidenced by SERRS along the $z$ axis, showing that the SERRS signal intensity of the molecule remained constant from the surface through $700 \mu \mathrm{m}$ in depth (Fig. 4e). A time-dependent (0, 8 and $24 \mathrm{~h}$ ) SERRS analysis of the diffusion of pyocyanin secreted by a growing biofilm shows homogeneous distribution of the molecule over the hydrogel surface. However, the diffusion is slightly compromised along the $z$ axis at $24 \mathrm{~h}$ of growth, most likely due to interferences caused by biofilm matrix components (Supplementary Fig. 4.2).

Current knowledge of the influence of QS in bacterial pathogenesis and biofilm development is largely derived from in vitro systems. However, as infection is a continuous interplay between the host and the microbes, experimental animal models are widely used in preclinical studies. Investigations of biofilmrelated infections in animals are often based on bacteria previously adhered to organic and inorganic materials, which are implanted in the animal ${ }^{37}$. For example, studies of chronic $P$. aeruginosa lung infection used embedded bacteria in natural hydrogels such as agar or alginate ${ }^{37,41}$. In vivo optical imaging combined with genetically modified bacteria expressing bioluminescent or fluorescent reporters is an effective method for performing longitudinal studies of bacterial infection in animal models ${ }^{42}$. Importantly, this imaging technique contributes towards the reduction in the number of experimental animals as it enables each animal to be analysed over time with no need for sequential euthanasia performed for ex vivo quantification of bacteria. However, optical imaging employing fluorescence or bioluminescence is often limited by autofluorescence and signal attenuation due to scattering and absorption of light by mammalian tissues ${ }^{42}$.

Since pyocyanin is detected by SERRS following irradiation with a near-infrared $(785 \mathrm{~nm})$ laser (within the biological transparency window), it can be used as a SERS beacon for reporting QS in infection studies employing plasmonic hydrogels pre-colonized 
with natural (for example, non-genetically modified) strains of P. aeruginosa. To test this idea, an Au@pNIPAM hydrogel was spiked with commercial pyocyanin at biologically relevant concentrations ${ }^{43}$ and the substrate was implanted in Swiss mice (Fig. 4f). As shown in the SERRS analysis of Fig. 4g, pyocyanin was detected subcutaneously at concentrations as low as $0.1 \mu \mathrm{M}$, suggesting that it could be used as a reporter for label-free, non-invasive monitoring of QS and screening potential antimicrobial drugs in animal models for infections, using SERS. P. aeruginosa is one of the most common bacteria isolated from soft tissue infections, such as those in acute burns, bed sores and diabetic ulcers ${ }^{9}$. P. aeruginosa infections are often characterized by increasing occurrence of multiresistance strains, leading to numerous deaths annually worldwide and thus cause a significant burden to healthcare systems. Hence, the development of new innovations towards their prevention and treatment has become imperative. Disruption of QS circuits with synthetic chemical compounds and natural products is a strategy with potential applications to prevent or attenuate bacterial pathogenesis ${ }^{44}$. Remarkably, the inhibition or decrease of pyocyanin expression is often used as an indicator of the treatment efficacy ${ }^{44-46}$ Thus, plasmonically active hydrogels could be furnished in standard animal models of wound infections ${ }^{47}$ as advanced in vivo optical sensors for providing new insights into the role of pyocyanin and QS in the pathogenesis of clinical strains of $P$. aeruginosa and to evaluate the efficacy of prophylactic and therapeutic treatments in longitudinal studies.

Microbial communities in biofilms are structurally organized entities, and the physical localization of signalling molecules within the cell population has profound impact on bacterial behaviour and phenotypic heterogeneity ${ }^{5,48}$. Therefore, the capacity to image the chemical environment surrounding biofilms with spatial resolution may provide new insights to understand their physiology and behaviour. We have demonstrated that Au@pNIPAM hydrogels allow non-invasive, in situ SERRS detection of pyocyanin expressed by growing colony-biofilms. However, the enhanced diffusion of this platform impairs the analysis of pyocyanin distribution over large sessile bacterial populations with meaningful spatial resolution. Mesostructured $\mathrm{Au} @ \mathrm{TiO}_{2}$ thin films were evaluated as substrates for spatially resolved plasmonic imaging of pyocyanin in biofilm communities of $P$. aeruginosa. The highly organized mesoporous nature of this platform (interpore distance $12 \mathrm{~nm}$; film thickness about $130 \mathrm{~nm}$ ) (Supplementary Fig. 1.2) was hypothesized to restrict horizontal diffusion of the analyte, so that localized detection of the phenazine can be achieved only at biofilmcolonized regions. In addition, the large area, averaging $1.0 \mathrm{~cm}^{2}$, allows plasmonic interrogation of pyocyanin expression over large (millimetre-scale) regions. A droplet-based assay was performed, in which a bacterial suspension $(50 \mu \mathrm{l})$ of $P$. aeruginosa PA14 $\left(1 \times 10^{9}\right.$ c.f.u. $\left.\mathrm{ml}^{-1}\right)$ depleted of extracellular pyocyanin was grown statically on $\mathrm{Au} @ \mathrm{TiO}_{2}$ at $30^{\circ} \mathrm{C}$ for $48 \mathrm{~h}$ inside a humidified chamber. The substrate allowed bacterial growth, as evidenced by the increased number of viable bacteria over time, as well as SERRS detection of pyocyanin exclusively in those regions of the mesoporous substrate in contact with the bacterial culture (Supplementary Fig. 4.3). Interestingly, the intensity of the SERRS signals was observed to increase towards the innermost regions of the drop, as evidenced by plotting the SERRS intensity $\left(418 \mathrm{~cm}^{-1}\right)$ as a function of distance (Supplementary Fig. 4.3). To correlate the presence of pyocyanin with biofilm bacteria, the substrate was gently immersed in water so as to reveal substrate-adhered cells. SERRS mapping spanning the region shown in Fig. 5a scanned with a $50 \mu \mathrm{m}$ step size demonstrated high-intensity pyocyanin SERRS signals at the central region, which was heavily colonized by bacteria as observed in the optical image acquired in situ. Significantly, the SERRS signal decreased towards less colonized regions and faded completely at non-colonized surfaces, indicating pyocyanin detection with a spatial resolution of about $50 \mu \mathrm{m}$. Analysis of the substrate by scanning electron microscopy (SEM) revealed the presence of bacterial microcolonies and self-produced extracellular polymeric substances (Fig. 5c and Supplementary Fig. 4.4), which are key features of biofilms. To further confirm the spatially resolved detection of pyocyanin, rather than growing the biofilm in a liquid environment, a small colony of $P$. aeruginosa PA14 strain was grafted on the Au@ $\mathrm{TiO}_{2}$ substrate, layered with a pad of $0.75 \%$ agarosecontaining LB medium and incubated for $24 \mathrm{~h}$. SERRS imaging performed in situ, without additional manipulation, revealed that pyocyanin signal was indeed restricted to the region colonized by bacteria (Supplementary Fig. 4.5).

In an effort to increase the sensitivity of this approach, micropatterned $\mathrm{Au} @ \mathrm{SiO}_{2}$ supercrystal arrays (Fig. 6a), which showed the highest enhancement factor towards pyocyanin detection (LOD $10^{-14} \mathrm{M}$ ) (Fig. 2d), were evaluated. Again, $10 \mu \mathrm{l}$ of a bacterial suspension $\left(1 \times 10^{9}\right.$ c.f.u. $\left.\mathrm{ml}^{-1}\right)$ devoid of pyocyanin was grown statically for 1,3 or $20 \mathrm{~h}$ on the patterned substrate and then analysed. Strikingly, the SERRS fingerprint of pyocyanin was identified as early as one hour after initiating the culture, and the intensity of the signal increased over time, as shown by the SERRS spectra recorded at 3 and $20 \mathrm{~h}$ (Fig. 6b,c). This indicates that starting with a cell density of approximately $1 \times 10^{7} \mathrm{cell} \mathrm{ml}^{-1}$, $P$. aeruginosa can soon engage in QS behaviour. When using this substrate we could also detect chloroform-extracted pyocyanin from a planktonic culture of $3.4 \times 10^{7}$ c.f.u. $\mathrm{ml}^{-1}$, which was not possible with either of the other two plasmonic substrates. This is reasonable, considering that the LOD of the $\mathrm{Au} @ \mathrm{SiO}_{2}$ substrate was shown to be 4 or 5 orders of magnitude lower than those of Au@pNIPAM and Au@ $\mathrm{TiO}_{2}$ substrates, respectively (Fig. 2d). Analysis of the substrate by SEM performed at three hours of growth provides evidence of the low cell density present in the bacterial culture (Supplementary Fig. 4.6a). SERRS mappings recorded after $20 \mathrm{~h}$ of growth demonstrated pyocyanin detection exclusively at the plasmonic features, $25 \mu \mathrm{m}^{2}$ on average (Fig. 6d). Inspection of the substrates by SEM revealed the presence of microcolonies and small groups of cells on top of the pedestals (Fig. 6e,f and Supplementary Fig. 4.6b,c), which should be responsible for the localized secretion of the phenazine. However, it cannot be ruled out that the SERRS signal may also arise from pyocyanin produced by nearby bacteria. This is in agreement with an earlier report based on the use of scanning electrochemical microscopy and a lithographic technique to confine small populations of $P$. aeruginosa bacteria in low volumes and reported that pyocyanin can be produced by clusters containing only 500 bacterial cells ${ }^{49}$.

\section{Outlook}

The engagement of bacteria in social behaviour, including the coordination of group activities via QS, such as biofilm formation, has become one of the most intensively studied topics in microbiology during the past few decades. In addition, the huge impact of bacterial biofilms in industrial fouling, contamination and infection has made this form of microbial growth a priority research topic. The strict QS control of pyocyanin expression, together with its high Raman cross-section, allowed us to use it as a beacon to demonstrate label-free plasmonic detection and imaging of QS communication in live biofilm communities of $P$. aeruginosa grown on three types of rationally designed SERS-active substrates. Au@pNIPAM hydrogels allowed proliferation of biofilms and homogeneous SERS sensing of the secreted phenazine across the substrate. The high water content and hydrophilic nature of hydrogels are similar to the void-filling component of microbial biofilms or the extracellular matrix of mammalian cells, which renders them intrinsically biocompatible. Thus, hydrogels are widely used in diverse biomedical applications as cellular scaffolds and bioactive materials ${ }^{50}$. For instance, hydrogels of controlled 
a

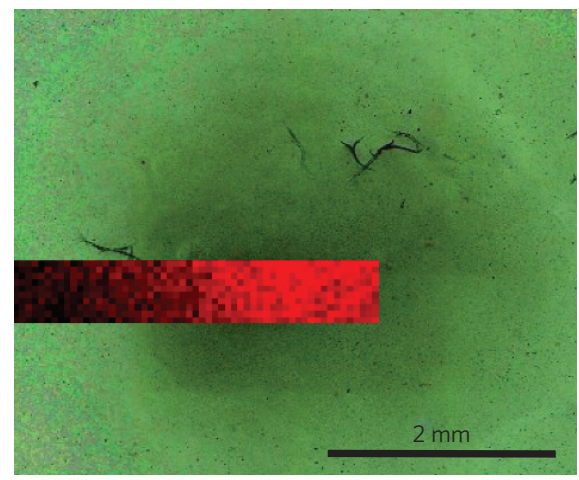

b

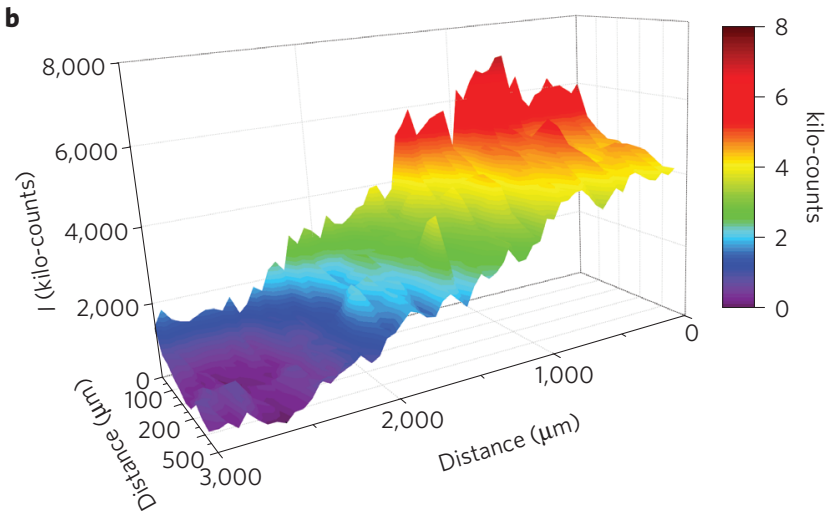

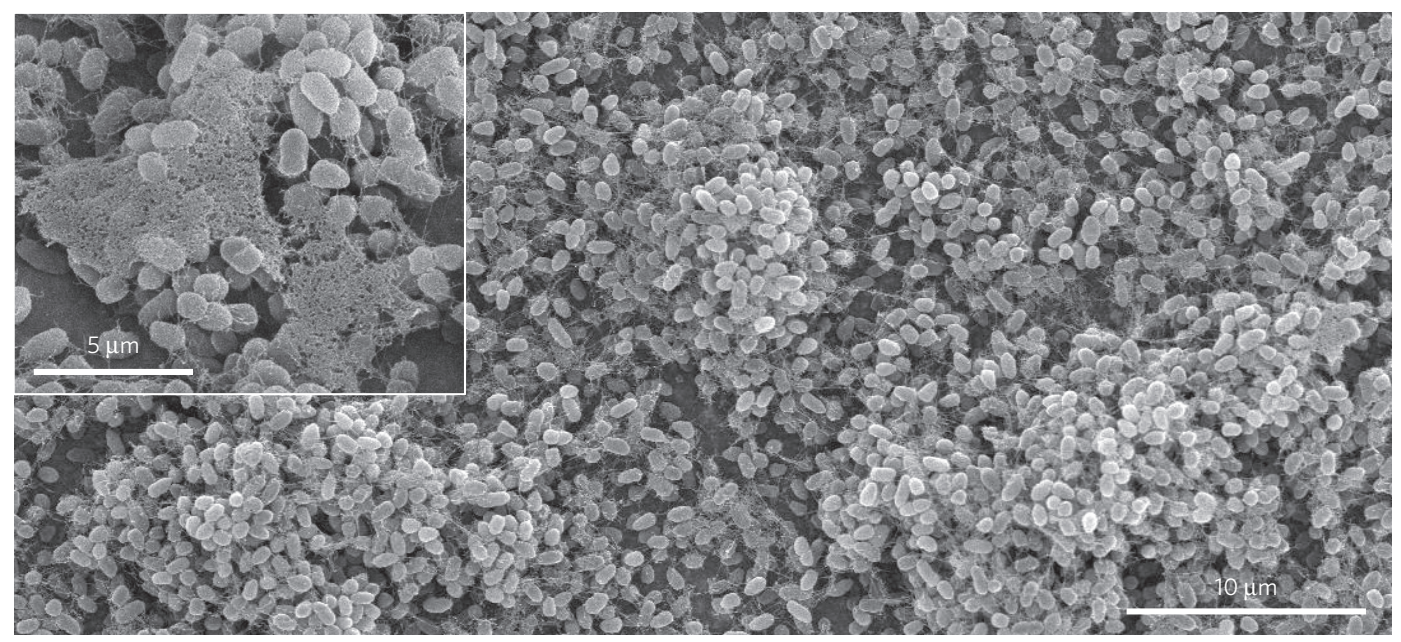

Figure 5 | In situ detection and imaging of pyocyanin produced by P. aeruginosa PA14 biofilms grown on mesostructured Au@TiO 2 thin films. a, Optical image of the bacterial biofilm (dark central region) captured with the Raman microscope and superimposed pyocyanin SERRS mapping $\left(418 \mathrm{~cm}^{-1}\right.$ ) acquired with excitation laser wavelength of $785 \mathrm{~nm}, 5 \times$ objective and a laser power of $0.94 \mathrm{~mW}$ for $10 \mathrm{~s}$. b. Graphical representation of the SERRS intensity mapping shown in a. c, Representative SEM images of bacterial biofilm formed on $\mathrm{Au}_{\mathrm{u}} \mathrm{TiO}_{2}$.

diffusivity and mass transfer properties have been fabricated by soft lithography for engineering cellular microenvironments in which cell populations can maintain chemical exchange and communication $^{48,50,51}$. Our results suggest that, besides providing scaffolding and cellular support, SERS-active nanostructured hydrogels could be implemented as plasmonic sensors for label-free characterization and monitoring of secreted diffusible biomolecules. Additionally, the results show that plasmonically active hydrogels can be used as implantable materials in experimental animal models, to investigate QS triggered by natural populations of $P$. aeruginosa and to evaluate anti-virulence therapies by pharmacologic intervention ${ }^{44}$.

Imaging QS in biofilm communities with spatial resolution is important towards gaining new understanding of this form of bacterial communication. It was demonstrated with mesostructured $\mathrm{Au} @ \mathrm{TiO}_{2}$ and micropatterned supercrystal $\mathrm{Au} @ \mathrm{SiO}_{2}$ platforms that $P$. aeruginosa not only engage QS in densely populated biofilms, but also at early stages of biofilm development. The mesostructured $\mathrm{Au} @ \mathrm{TiO}_{2}$ platform enabled us to interrogate up to millimetre-scale regions of the colonized substrate with high spatial resolution $(20-50 \mu \mathrm{m})$. The micropatterned supercrystal $\mathrm{Au} @ \mathrm{SiO}_{2}$ enabled ultrasensitive SERS detection of QS events in low-density bacterial cultures at the initial hours of growth and imaging of bacterial communication, owing to its high enhancement factor. The $\mathrm{Au} @ \mathrm{SiO}_{2}$ substrates exhibited pyocyanin detection down to $10^{-14} \mathrm{M}$, possibly triggered by small clusters of cells colonizing the micrometre-sized plasmonic features. The high performance of this substrate is most likely due to a high density of efficient hotspots and collective plasmon modes in the supercrystal ${ }^{35}$. Since it is well known that the electric field enhancement decays very rapidly from the plasmonic nanoparticles surface $^{52}$, it is essential to consider that the mesoporous silica coating infiltrates within the highly ordered nanorod structure ${ }^{53}$, thereby increasing the 'plasmonically active space' and improving the sensitivity of the Au@SiO${ }_{2}$ optical enhancer. Microfabrication of ordered plasmonic nanoparticle supercrystals provides unique optical and photonic coupling effects but also patterning is an interesting strategy for fabricating complex nanostructured sensing materials. For example, micropatterning mediated by nano- and microfabrication techniques has been applied to the analysis of cell behaviour on substrates with ordered topographical features ${ }^{54-56}$. Thus, the ability to selectively immobilize cells and/or modulate cellular responses at plasmonically active micropatterned features, combined with ultrasensitive SERS detection, will provide powerful analytical platforms for providing more insight into cell-to-cell communication in microenvironments based on diffusible SERSactive signals.

The high resolution displayed by the $\mathrm{Au} @ \mathrm{TiO}_{2}$ and $\mathrm{Au} @ \mathrm{SiO}_{2}$ plasmonic substrates $(20-50 \mu \mathrm{M})$ is in the range of that obtained with most imaging mass spectrometry (IMS) techniques $(1-100 \mu \mathrm{m})$, which have been successfully applied to probe single cells and colonies in microbial research ${ }^{57,58}$. A key advantage of IMS is that it enables the label-free spatial imaging of the distribution of biomolecules in biological samples. However, IMS techniques often require various forms of sample preparation that may disturb the specimen under investigation (for example, 
a

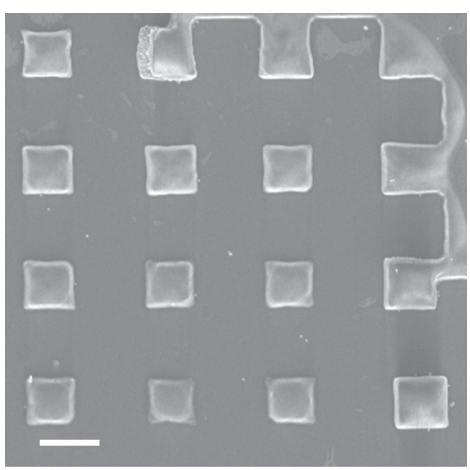

d

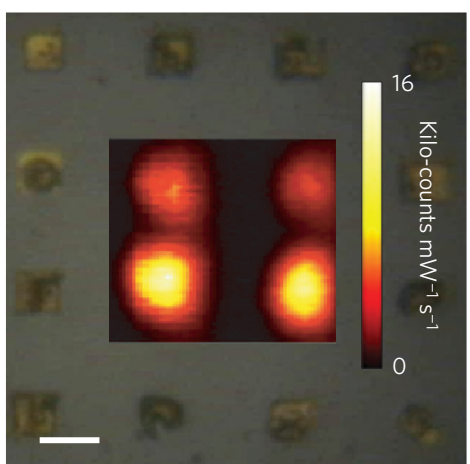

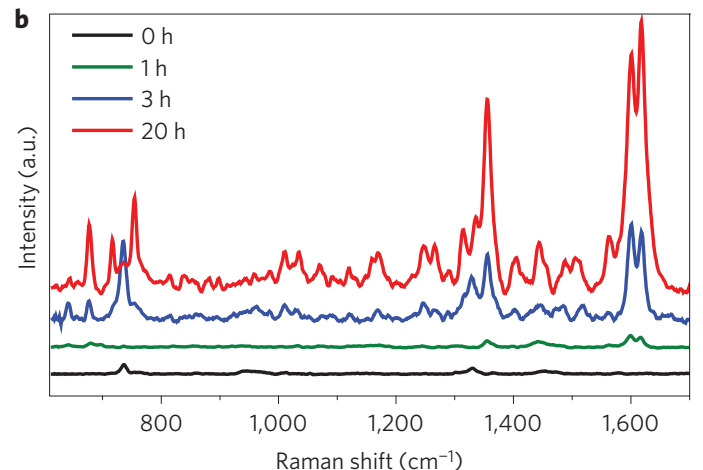

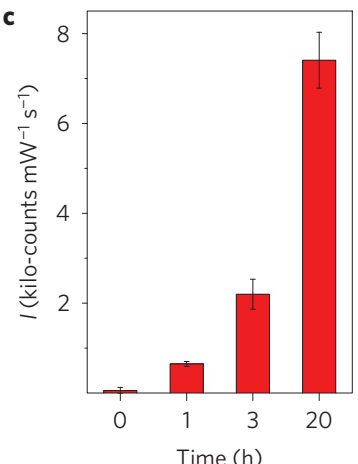

e

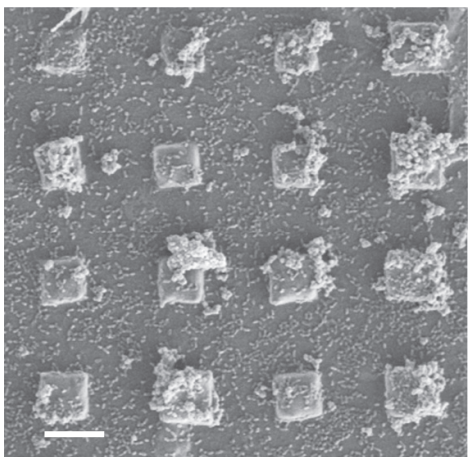

f

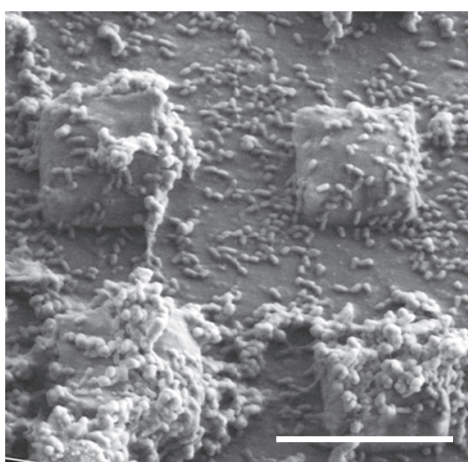

Figure 6 | In situ detection and imaging of pyocyanin produced by P. aeruginosa PA14 grown on micropatterned Au@SiO $\mathbf{2}_{\mathbf{2}}$ substrates. a, SEM images of silica-coated Au nanorod supercrystals. Scale bar, $5 \mu \mathrm{m}$. b. Representative SERRS spectra measured at 0, 1, 3 and 20 h. c, Relative SERRS intensities $\left(1,600 \mathrm{~cm}^{-1}\right)$ recorded at $0,1,3$ and $20 \mathrm{~h}$. Error bars indicate standard deviation. d, Optical image of the substrate and SERRS mapping of pyocyanin $\left(1,600 \mathrm{~cm}^{-1}\right)$ recorded at $20 \mathrm{~h}$ of growth. Scale bar, $5 \mu \mathrm{m}$. e,f, SEM images of silica-coated Au nanorod supercrystals colonized by $P$. aeruginosa $(20 \mathrm{~h})$ at different magnifications. Scale bars, $5 \mu \mathrm{m}$. All SERS measurements were carried out with a $785 \mathrm{~nm}$ laser line, $50 \times$ objective and a maximum power of $0.98 \mathrm{~kW} \mathrm{~cm}^{-2}$. The acquisition time was $0.1 \mathrm{~s}$.

layering with an inorganic compound in matrix-assisted laser desorption/ionization, or coating with an organic matrix in matrixassisted laser desorption/ionization-time of flight), as well as the limited mass-resolving power of $\mathrm{IMS}^{57}$. As we have shown, our SERS-based approach, focusing on the detection of pyocyanin released from bacterial biofilms and small clusters of cells, demonstrates the potential of plasmonics as an alternative for imaging biomolecules from undisturbed biological samples. The porous nature of the substrates plays an important role in restricting the contact of the plasmonic component with (bio)molecules that could contaminate the SERS spectrum and thereby hinder the selectivity and sensitivity of the assay. Large molecules may also contaminate the plasmonic surface and passivate it from interaction with the analyte. The SERS-based sensing approaches described in this work not only provide the necessary tools to probe important questions in QS, but can also serve as general detection systems to be applied for the investigation of other cellular communication processes based on SERS-active diffusible molecules.

\section{Methods}

Methods and any associated references are available in the online version of the paper.

Received 28 January 2016; accepted 4 July 2016; published online 8 August 2016

\section{References}

1. Waters, C. M. \& Bassler, B. L. Quorum sensing: cell-to-cell communication in bacteria. Annu. Rev. Cell Dev. Biol. 21, 319-346 (2005).

2. Lazdunski, A. M., Ventre, I. \& Sturgis, J. N. Regulatory circuits and communication in gram-negative bacteria. Nature Rev. Microbiol. 2, 581-592 (2004).
3. Rutherford, S. T. \& Bassler, B. L. Bacterial quorum sensing: its role in virulence and possibilities for its control. Cold Spring Harb. Perspect. Med. 2, a012427 (2012).

4. LaSarre, B. \& Federle, M. J. Exploiting quorum sensing to confuse bacterial pathogens. Microbiol. Mol. Biol. Rev. 77, 73-111 (2013).

5. Hall-Stoodley, L., Costerton, J. W. \& Stoodley, P. Bacterial biofilms: from the natural environment to infectious diseases. Nature Rev. Microbiol. 2, 95-108 (2004)

6. Davey, M. E. \& O’toole, G. A. Microbial biofilms: from ecology to molecular genetics. Microbiol. Mol. Biol. Rev. 64, 847-867 (2000).

7. Li, Y. H. \& Tian, X. L. Quorum sensing and bacterial social interactions in biofilms. Sensors 12, 2519-2538 (2012).

8. Parsek, M. R. \& Greenberg, E. P. Sociomicrobiology: the connections between quorum sensing and biofilms. Trends Microbiol. 13, 27-33 (2005).

9. Rybtke, M., Hultqvist, L. D., Givskov, M. \& Tolker-Nielsen, T. Pseudomonas aeruginosa biofilm infections: community structure, antimicrobial tolerance and immune response. J. Mol. Biol. 427, 3628-3645 (2015).

10. Jimenez, P. N. et al. The multiple signaling systems regulating virulence in Pseudomonas aeruginosa. Microbiol. Mol. Biol. Rev. 76, 46-65 (2012).

11. Castillo-Juarez, I. et al. Role of quorum sensing in bacterial infections. World J Clin. Cases 3, 575-598 (2015).

12. Decho, A. W., Norman, R. S. \& Visscher, P. T. Quorum sensing in natural environments: emerging views from microbial mats. Trends Microbiol. 18, 73-80 (2010).

13. Steindler, L. \& Venturi, V. Detection of quorum-sensing N-acyl homoserine lactone signal molecules by bacterial biosensors. FEMS Microbiol. Lett. 266, 1-9 (2007).

14. Jansson, J. K. Marker and reporter genes: illuminating tools for environmental microbiologists. Curr. Opin. Microbiol. 6, 310-316 (2003).

15. Moskovits, M. Surface-enhanced Raman spectroscopy: a brief retrospective. J. Raman Spectrosc. 36, 485-496 (2005).

16. Stiles, P. L., Dieringer, J. A., Shah, N. C. \& Van Duyne, R. R. Surface-enhanced Raman spectroscopy. Annu. Rev. Anal. Chem. 1, 601-626 (2008).

17. Schlucker, S. Surface-enhanced Raman spectroscopy: concepts and chemical applications. Angew. Chem. Int. Ed. 53, 4756-4795 (2014).

18. Anker, J. N. et al. Biosensing with plasmonic nanosensors. Nature Mater. 7, 442-453 (2008) 
19. Bantz, K. C. et al. Recent progress in SERS biosensing. Phys. Chem. Chem. Phys. 13, 11551-11567 (2011).

20. Abalde-Cela, S. et al. Surface-enhanced Raman scattering biomedical applications of plasmonic colloidal particles. J. R. Soc. Interface 7, S435-S450 (2010).

21. Alvarez-Puebla, R. A. \& Liz-Marzan, L. M. SERS-based diagnosis and biodetection. Small 6, 604-610 (2010).

22. Howes, P. D., Chandrawati, R. \& Stevens, M. M. Colloidal nanoparticles as advanced biological sensors. Science 346, 1247390 (2014).

23. Zeng, S. W., Baillargeat, D., Ho, H. P. \& Yong, K. T. Nanomaterials enhanced surface plasmon resonance for biological and chemical sensing applications. Chem. Soc. Rev. 43, 3426-3452 (2014).

24. Pierson, L. S. \& Pierson, E. A. Metabolism and function of phenazines in bacteria: impacts on the behavior of bacteria in the environment and biotechnological processes. Appl. Microbiol. Biotechnol. 86, 1659-1670 (2010).

25. Mavrodi, D. V. et al. Functional analysis of genes for biosynthesis of pyocyanin and phenazine-1-carboxamide from Pseudomonas aeruginosa PAO1. J. Bacteriol. 183, 6454-6465 (2001).

26. Dietrich, L. E. P. et al. The phenazine pyocyanin is a terminal signalling factor in the quorum sensing network of Pseudomonas aeruginosa. Mol. Microbiol. 61, 1308-1321 (2006).

27. Dietrich, L. E. P., Teal, T. K., Price-Whelan, A. \& Newman, D. K. Redox-active antibiotics control gene expression and community behavior in divergent bacteria. Science 321, 1203-1206 (2008).

28. Ramos, I., Dietrich, L. E. P., Price-Whelan, A. \& Newman, D. K. Phenazines affect biofilm formation by Pseudomonas aeruginosa in similar ways at various scales. Res. Microbiol. 161, 187-191 (2010).

29. Dietrich, L. E. P. et al. Bacterial community morphogenesis is intimately linked to the intracellular redox state. J. Bacteriol. 195, 1371-1380 (2013).

30. Price-Whelan, A., Dietrich, L. E. \& Newman, D. K. Rethinking 'secondary' metabolism: physiological roles for phenazine antibiotics. Nature Chem. Biol. 2, 71-78 (2006).

31. Lee, J. \& Zhang, L. H. The hierarchy quorum sensing network in Pseudomonas aeruginosa. Protein Cell 6, 26-41 (2015).

32. Wu, X. M. et al. Culture-free diagnostics of Pseudomonas aeruginosa infection by silver nanorod array based SERS from clinical sputum samples. Nanomedicine 10, 1863-1870 (2014).

33. Lopez-Puente, V. et al. Plasmonic mesoporous composites as molecular sieves for SERS detection. J. Phys. Chem. Lett. 4, 2715-2720 (2013).

34. Yang, S., Dai, X., Boschitsch Stogin, B. \& Wong, T. Ultrasensitive surface-enhanced Raman scattering detection in common fluids. Proc. Natl Acad. Sci. USA 113, 268-273 (2015).

35. Hamon, C. et al. Hierarchical self-assembly of gold nanoparticles into patterned plasmonic nanostructures. ACS Nano 8, 10694-10703 (2014).

36. Reszka, K. J. et al. Oxidation of pyocyanin, a cytotoxic product from Pseudomonas aeruginosa, by microperoxidase 11 and hydrogen peroxide. Free Radic. Biol. Med. 36, 1448-1459 (2004).

37. Lebeaux, D., Chauhan, A., Rendueles, O. \& Beloin, C. From in vitro to in vivo models of bacterial biofilm-related infections. Pathogens 2, 288-356 (2013).

38. Coenye, T. \& Nelis, H. J. In vitro and in vivo model systems to study microbia biofilm formation. J. Microbiol. Methods 83, 89-105 (2010).

39. Koley, D., Ramsey, M. M., Bard, A. J. \& Whiteley, M. Discovery of a biofilm electrocline using real-time 3D metabolite analysis. Proc. Natl Acad. Sci. USA 108, 19996-20001 (2011).

40. Bellin, D. L. et al. Integrated circuit-based electrochemical sensor for spatially resolved detection of redox-active metabolites in biofilms. Nature Commun. 5, 3256 (2014).

41. Pedersen, S. S., Shand, G. H., Hansen, B. L. \& Hansen, G. N. Induction of experimental chronic Pseudomonas-aeruginosa lung infection with Pseudomonas-aeruginosa entrapped in alginate microspheres. APMIS 98, 203-211 (1990).

42. Leevy, W. M., Serazin, N. \& Smith, B. D. Optical imaging of bacterial infection models. Drug Discov. Today Dis. Models 4, 91-97 (2007).

43. Hunter, R. C. et al. Phenazine content in the cystic fibrosis respiratory tract negatively correlates with lung function and microbial complexity. Am. J. Respir. Cell Mol. Biol. 47, 738-745 (2012).
44. Reuter, K., Steinbach, A. \& Helms, V. Interfering with bacterial quorum sensing. Perspect. Medicin. Chem. 8, 1-15 (2016).

45. O'Loughlin, C. T. et al. A quorum-sensing inhibitor blocks Pseudomonas aeruginosa virulence and biofilm formation. Proc. Natl Acad. Sci. USA 110, 17981-17986 (2013).

46. Welsh, M. A., Eibergen, N. R., Moore, J. D. \& Blackwell, H. E. Small molecule disruption of quorum sensing cross-regulation in Pseudomonas aeruginosa causes major and unexpected alterations to virulence phenotypes. J. Am. Chem. Soc. 137, 1510-1519 (2015).

47. Dai, T. H. et al. Animal models of external traumatic wound infections. Virulence 2, 296-315 (2011).

48. Wessel, A. K., Hmelo, L., Parsek, M. R. \& Whiteley, M. Going local: technologies for exploring bacterial microenvironments. Nature Rev. Microbiol. 11, 337-348 (2013).

49. Connell, J. L. et al. Real-time monitoring of quorum sensing in 3D-printed bacterial aggregates using scanning electrochemical microscopy. Proc. Natl Acad. Sci. USA 111, 18255-18260 (2014).

50. Buenger, D., Topuz, F. \& Groll, J. Hydrogels in sensing applications. Prog. Polym. Sci. 37, 1678-1719 (2012).

51. Weibel, D. B., DiLuzio, W. R. \& Whitesides, G. M. Microfabrication meets microbiology. Nature Rev. Microbiol. 5, 209-218 (2007).

52. Tian, L. et al. Plasmonic biofoam: a versatile optically active material. Nano Lett. 16, 609-616 (2016).

53. Hamon, C. et al. Hierarchical organization and molecular diffusion in gold nanorod/silica supercrystal nanocomposites. Nanoscale 8, 7914-7922 (2016).

54. Falconnet, D., Csucs, G., Grandin, H. M. \& Textor, M. Surface engineering approaches to micropattern surfaces for cell-based assays. Biomaterials 27, 3044-3063 (2006).

55. Shao, Y. \& Fu, J. P. Integrated micro/nanoengineered functional biomaterials for cell mechanics and mechanobiology: a materials perspective. Adv. Mater. 26, 1494-1533 (2014)

56. Mitragotri, S. \& Lahann, J. Physical approaches to biomaterial design. Nature Mater. 8, 15-23 (2009).

57. Watrous, J. D. \& Dorrestein, P. C. Imaging mass spectrometry in microbiology. Nature Rev. Microbiol. 9, 683-694 (2011).

58. Fang, J. S. \& Dorrestein, P. C. Emerging mass spectrometry techniques for the direct analysis of microbial colonies. Curr. Opin. Microbiol. 19, 120-129 (2014).

\section{Acknowledgements}

This work has been funded by the European Research Council (ERC Advanced Grant no. 267867 Plasmaquo). We thank D. K. Newman, Department of Biology, California Institute of Technology and Howard Hughes Medical Institute for providing us with P. aeruginosa PA14 and PA14 $\Delta p h z 1 / 2$ strains. We thank F. M. Ausubel, Department of Genetics, Harvard Medical School for providing P. aeruginosa PA14 phzH, phzM and $p h z S$ strains. E. Modin and A. Chuvilin are thanked for their work on FIB/SEM of supercrystals. E.H.H. gratefully acknowledges the Spanish Ministry of Economy and Competitiveness for funding a Juan de la Cierva Fellowship. V.M.-G. acknowledges an FPU scholarship from the Spanish MINECO.

\section{Author contributions}

G.B. designed and executed bacterial growth experiments, V.M.-G. performed SERS experiments with Au@pNIPAM substrates, V.L.-P. fabricated $\mathrm{Au} @ \mathrm{TiO}_{2}$ substrates and measured SERS, C.C. executed bacterial growth experiments, S.R.-C. and S.C. participated in the fabrication of Au@pNIPAM substrates, I.P.-J. carried out DFT calculations, E.H.H. executed bacterial growth experiments and measured SERS on $\mathrm{Au} @ \mathrm{SiO}_{2}$ substrates, C.H., M.N.S.-O. and L.S. participated in the fabrication of $\mathrm{Au} @ \mathrm{SiO}_{2}$ substrates, A.L.P. carried out SEM imaging, I.P.-S. and J.P.-J. contributed to substrate design and discussion, L.M.L.-M. designed and supervised the project and the discussions. All authors participated in writing of the manuscript.

\section{Additional information}

Supplementary information is available in the online version of the paper. Reprints and permissions information is available online at www.nature.com/reprints.

Correspondence and requests for materials should be addressed to G.B. or L.M.L.-M.

\section{Competing financial interests}

The authors declare no competing financial interests. 


\section{Methods}

Materials. Tetrachloroauric(III) acid trihydrate $\left(\mathrm{HAuCl}_{4} \cdot 3 \mathrm{H}_{2} \mathrm{O}\right)$, L-ascorbic acid (ACS reagent 99\%), $\mathrm{N}$-isopropylacrylamide (NIPAM, 97\%),

$N, N^{\prime}$-methylenebis(acrylamide) (BIS, 99\%),

$N, N, N^{\prime}, N^{\prime}$-tetramethylethylenediamine (TEMED), 5-bromosalicylic acid (technical grade $90 \%$ ), silver nitrate $\left(\mathrm{AgNO}_{3}\right.$, ACS reagent $\left.99.0 \%\right)$, sodium borohydride $\left(\mathrm{NaBH}_{4}\right.$, ReagentPlus $\left.99 \%\right)$, O-[2-(3-mercaptopropionylamino)ethyl] - $\mathrm{O}^{\prime}$-methyl-poly(ethylene glycol) (mPEG-SH, Mw 5,000), trisodium citrate dihydrate, titanium tetrachloride ( $\left.\mathrm{TiCl}_{4}\right),(3$-aminopropyl)trimethoxysilane (APS), Pluronic F127 $\left(\mathrm{HO}\left(\mathrm{CH}_{2} \mathrm{CH}_{2} \mathrm{O}\right)_{106}\left(\mathrm{CH}_{2} \mathrm{CH}\left(\mathrm{CH}_{3}\right)-\mathrm{O}\right)_{70}\left(\mathrm{CH}_{2} \mathrm{CH}_{2} \mathrm{O}\right)_{106} \mathrm{OH}\right)$, hydrogen peroxide $\left(\mathrm{H}_{2} \mathrm{O}_{2}, 28 \%\right)$, sulfuric acid $\left(\mathrm{H}_{2} \mathrm{SO}_{4}, 98 \%\right)$, chloroform $(99.8 \%)$ and pyocyanin from Pseudomonas aeruginosa were supplied by Sigma Aldrich. Hydrochloric acid ( $\mathrm{HCl}, 37 \mathrm{wt} \%$ in water) was supplied by Panreac. Cetyltrimethylammonium bromide (CTAB, 96\%) was supplied by Fluka. All chemicals were used as received. Pure-grade ethanol and Milli-Q water were used as solvents.

Characterization. Optical characterization was carried out by UV-visible-near-infrared spectroscopy with a Cary 5000 spectrophotometer and an Agilent 8453 spectrophotometer. Transmission electron microscopy (TEM) analysis was performed by using a JEOL JEM 1010 microscope operating at an

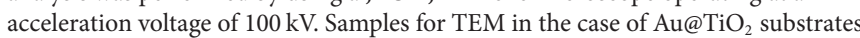
were obtained by scratching the films from the substrate and depositing them on carbon- and FORMVAR-coated copper grids. The surface morphology of the dried gels was investigated by using a scanning electron microscope (JEOL JSM-6700F FEG).

Raman spectroscopy experiments were conducted with a Renishaw InVia Reflex system. The spectrograph used a high-resolution grating ( 1,200 or 1,800 grooves $\mathrm{mm}^{-1}$ ) with additional band-pass filter optics, a confocal microscope and a two-dimensional CCD (charge-coupled device) camera. Several laser excitation energies were employed, including laser lines at $633(\mathrm{HeNe})$ and 785 and $830 \mathrm{~nm}$ (diode)

Scanning electron microscopy (SEM) analysis was performed with a JEOL JSM-6700F or with a Helios NanoLab 450S scanning electron microscopes.

Fabrication of the plasmonic substrates. Au@pNIPAM hydrogels (1 mm thickness) were prepared by free-radical crosslinking copolymerization of $\mathrm{N}$-isopropylacrylamide and bisacrylamide (as crosslinker) in an aqueous solution containing PEGylated Au nanorods ${ }^{59}$. A solution of $1 \mathrm{ml}$ of the monomer NIPAM $(0.85 \mathrm{M})$, crosslinker BIS $(4.24 \mathrm{mM})$ and APS $(0.085 \mathrm{M})$ as the initiator was purged with nitrogen to remove the dissolved oxygen in the system. Subsequently, a $0.5 \mathrm{ml}$ dispersion of gold nanorods (average dimensions: $91.3 \pm 8.9 \mathrm{~nm}$ long; $27.0 \pm 2.3 \mathrm{~nm}$ thick $)^{60}$ functionalized with mPEG-SH $\left(5\right.$ molecules $\left.\mathrm{nm}^{-2}\right)$ and accelerator TEMED $(1.5 \mu \mathrm{l})$ were mixed with the solution, and were quickly transferred to a mould consisting of two glass plates separated by 1.0 -mm-thick spacers. Polymerization was carried out at room temperature for $1 \mathrm{~h}$. The resulting hydrogel was removed from the mould, and gels were cut into pieces of $1 \mathrm{~cm}^{2}$. The gels pieces were washed extensively in an excess of deionized water. The water was replaced every day to remove the residual unreacted components and the washing was continued for at least 3 days.

The optical characterization of the Au@pNIPAM substrate reveals that the hydrogel retains the main optical features of the Au nanorods indicating uniform nanoparticle distribution (Supplementary Fig. 1.1.a), which was also confirmed by SEM analysis (Supplementary Fig. 1.1.b).

Mesostructured $\mathrm{Au} @ \mathrm{TiO}_{2}$ substrates were synthesized depositing a mesoporous thin film on a submonolayer of $80 \mathrm{~nm}$ Au nanospheres by spin-coating using block copolymer Pluronic F127 micelles as templates ${ }^{33}$. This procedure leads to highly ordered body-centred cubic $(\operatorname{Im} 3 \mathrm{~m})$ pore system, which is commonly obtained when templating with $\mathrm{F} 127^{61}$. The visible-near-infrared spectrum of the $\mathrm{Au} @ \mathrm{TiO}_{2}$ substrate (Supplementary Fig. 1.1.c) shows the presence of two bands, one at $580 \mathrm{~nm}$ from the single particles and a broad band at around $830 \mathrm{~nm}$ due to plasmon coupling. As can be observed in Supplementary Fig. 1.1.d, the TEM image of the composite substrate shows ordered mesoporous structures over the metal nanoparticles. The pore diameter and wall thickness (that is, interpore distance) measured from TEM images yielded average values of $12 \mathrm{~nm}$, and the film thickness measured from SEM cross-section images led to average values of $130 \pm 6 \mathrm{~nm}$ (Supplementary Fig. 1.2).

For the micropatterned $\mathrm{Au} @ \mathrm{SiO}_{2}$ supercrystal arrays, the microtextured mould for the supercrystals was manufactured in polydimethylsiloxane (PDMS; Sylgard 184, Dow Corning), following standard soft lithography techniques ${ }^{62,63}$. The master was composed of pillars having different shapes and sizes on a 4 inch silicon wafer covered with SU-8 resin. PDMS (10:1 elastomer to curing agent) was cured at $60^{\circ} \mathrm{C}$ for $2 \mathrm{~h}$. In this work, assembly experiments were performed on $\langle 100\rangle$ silicon wafers.

For GNR supercrystal assembly, patterned substrates were fabricated by drying a GNR dispersion within an array of micrometre-sized cavities with a fixed height of $4.8 \mu \mathrm{m}$ and varying lateral dimensions and shapes, as previously reported ${ }^{35}$. By adjusting the cavity morphology of the template together with the particle concentration, the morphology and height of the supercrystals can be tuned. In all cases, a $2 \mu \mathrm{l}$ drop of MUDOL-GNR dispersion with the selected concentration was deposited on the microtextured PDMS template and then covered by the substrate. The solvent was subsequently allowed to evaporate to dryness (within $12 \mathrm{~h}$ ) and the template was removed resulting in the formation of arrays of GNR supercrystals spread over millimetre-sized areas.

For the silica coating procedure, before silica growth, the substrates were cleaned with a combination of oxygen plasma ( $2 \mathrm{~min}, 0.4 \mathrm{mbar}, 200 \mathrm{~W}$ ) and UV/ozone (1 h). Oxygen plasma surface treatment was performed in a low-pressure plasma system (PICO, Diener Electronic). UV/ozone surface treatment was performed in a UV/Ozone Cleaner (ProCleaner).

For the mesoporous silica film growth, $17.5 \mathrm{ml}$ of a $6 \mathrm{mM}$ CTAB solution in water was mixed with $7.5 \mathrm{ml}$ of ethanol and stirred at $35^{\circ} \mathrm{C}$ for $10 \mathrm{~min}$. Five microlitres of $\mathrm{NH}_{3}(25 \mathrm{vol} \%)$ was added to achieve a pH value between 9 and 10 . Finally, $20 \mu \mathrm{l}$ of TEOS was added drop-wise while stirring vigorously. One-millilitre aliquots of growth solution were immediately poured into Eppendorf centrifuge tubes, where a clean gold substrate had previously been introduced in each of the tubes. The tubes were subsequently closed and sealed with Parafilm to avoid evaporation of the synthesis solution and placed in an oven to incubate at $60^{\circ} \mathrm{C}$ for three days. The GNR silica substrates were then rinsed with ethanol and immersed in a $0.1 \mathrm{M} \mathrm{HCl}$ solution (in ethanol) for $10 \mathrm{~min}$ to get rid of excess CTAB. A further rinsing step in ethanol was performed to remove any trace of $\mathrm{HCl}$. The films were then treated at $130^{\circ} \mathrm{C}$ for $2 \mathrm{~h}$ to improve silica stability in water without significant reshaping of the nanorods. SEM images of the silica-coated composite substrate (Supplementary Fig. 1.3) show the internal nanorod organization and the presence of mesoporous silica infiltrating nanorod monolayers.

Analysis of the SERS performance of the plasmonic substrates. Au@pNIPAM hydrogels.An Au@pNIPAM hydrogel was immersed overnight in an aqueous solution of 1-naphthalenethiol (1-NAT) and SERS spectra were recorded under excitation with three different laser lines $(633,785$ and $803 \mathrm{~nm})$, obtaining in all cases well-defined bands characteristic of 1-NAT vibrations. SERS mapping additionally demonstrated that the SERS enhancing ability of hydrogel is uniform over extended areas (Supplementary Fig. 2.1). We therefore conclude that Au@pNIPAM hydrogels are efficient SERS substrates offering a wide excitation wavelength range.

Mesostructured Au@TiO $\mathrm{T}_{2}$ substrates. The efficiency of $\mathrm{Au} @ \mathrm{TiO}_{2}$ mesoporous thin films was confirmed using 4-nitrobenzenethiol (4-NBT) as analyte (data not shown), as well as its ability to exclude large biomolecules from the interior of the mesoporous films when analysing complex matrices (that is, molecular sieve effect), as previously reported ${ }^{33}$.

Micropatterned $\mathrm{Au} @ \mathrm{SiO}_{2}$ supercrystal arrays. The efficiency of micropatterned $\mathrm{Au} @ \mathrm{SiO}_{2}$ supercrystal arrays was tested using commercial pyocyanin as analyte. The SERS spectrum was recorded under a $785 \mathrm{~nm}$ laser line obtaining well-defined bands characteristic of pyocyanin vibrations. SERS mapping additionally demonstrated that the SERS enhancing ability of the supercrystals is uniform and confined to the supercrystal (Supplementary Fig. 2.2).

Raman enhancement factors calculations. We estimated the enhancement factors (EFs) of the different plasmonic substrates by applying the following equation ${ }^{64}$ :

$$
\mathrm{EF}=\left(I_{\mathrm{A}} V_{\mathrm{A}} / I_{\mathrm{B}} V_{\mathrm{B}}\right) \times f
$$

where $V_{\mathrm{A}}$ and $V_{\mathrm{B}}$ represent the probed volumes, and $I_{\mathrm{A}}$ and $I_{\mathrm{B}}$ the intensities in SERS and Raman, respectively; $f$ is a correction factor that considers the concentration ratio of the probed molecule in both experiments under the same conditions. Since pyocyanin is a solid, Raman spectra were collected from a $10^{-4} \mathrm{M}$ solution. Provided that $V_{\mathrm{A}}$ and $V_{\mathrm{B}}$ are similar, equation (1) can be reduced to $\mathrm{EF}=\left(I_{\mathrm{A}} / I_{\mathrm{B}}\right) \times f$, where $f$ is 5,4 and 6 for the Au@pNIPAM hydrogels, the $\mathrm{Au} @ \mathrm{TiO}_{2}$ mesostructured substrate and the micropatterned $\mathrm{Au} @ \mathrm{SiO}_{2}$ supercrystal array, respectively. For a reliable estimation of the EF we have considered the SERS intensity of the lowest concentration of pyocyanin within the quantitative detection region.

The estimated EFs are $3.99 \times 10^{5}, 1.55 \times 10^{6}$ and $1.67 \times 10^{6}$ for the Au@pNIPAM hydrogels, the $\mathrm{Au} @ \mathrm{TiO}_{2}$ mesostructured substrate and the micropatterned $\mathrm{Au@SiO}$, supercrystal array, respectively. The calculated EF can be considered as high since pyocyanin does not contain any thiol or amine functional group, so that its affinity for gold should be low.

Plasmonic detection of pyocyanin in planktonic and biofilm cultures. Bacterial strains and growth conditions of planktonic cultures. PA14 Pseudomonas aeruginosa bacterial strains used are indicated in Supplementary Table 4.1. Bacteria were streaked onto Luria-Bertani (LB) agar $(1.5 \% \mathrm{w} / \mathrm{v})$ plates and incubated overnight at $37^{\circ} \mathrm{C}$. A single colony was used to inoculate a $10 \mathrm{ml}$ culture in $\mathrm{LB}$ medium and 
grown at $37^{\circ} \mathrm{C}$ with agitation (210 r.p.m.). The attenuance $(D)$ of the liquid culture was measured at $D_{600 \mathrm{~nm}}$ for monitoring bacterial growth.

Extraction of phenazines and spectrophotometric quantification. Cells from a bacterial culture grown in LB were pelleted by centrifugation $(8,000 \mathrm{~g} / 3 \mathrm{~min})$ and the supernatant was filtered $(0.2 \mu \mathrm{m}$ filter pore size). The filtrate $(2 \mathrm{ml})$ was mixed thoroughly with the $4 \mathrm{ml}$ of chloroform and incubated for $20 \mathrm{~min}$, after which the aqueous phase was removed. Phenazines were recovered by air- or nitrogen-drying the solvent and re-dispersion in $2 \mathrm{ml}$ of water. The concentration of pyocyanin was determined spectrophotometrically on the basis of its absorbance at $691 \mathrm{~nm}$ $\left(A_{691 \mathrm{~nm}}\right)$, according to the Beer-Lambert law, knowing the $\varepsilon_{\mathrm{PYO}}=4,310 \mathrm{M}^{-1} \mathrm{~cm}^{-1}$ (ref. 65).

SERS detection of pyocyanin in planktonic cultures. Bacteria-free supernatants of P. aeruginosa PA14 obtained from a late stationary-phase culture grown for $20 \mathrm{~h}$ were incubated for at least five hours with the different plasmonic substrates. In the case of Au@pNIPAM hydrogels 1:100 dilution in water of the filtered supernatant was performed to reduce interference (SERS background), whereas this was not necessary in the mesostructured $\mathrm{Au} @ \mathrm{TiO}_{2}$ and micropatterned $\mathrm{Au} @ \mathrm{SiO}_{2}$ substrates, possibly due to the molecular sieve effect of their mesoporous layer ${ }^{33}$.

SERS detection of pyocyanin in biofilms grown on plasmonic substrates. The Au@pNIPAM hydrogel was sterilized under an ultraviolet light lamp for $16 \mathrm{~h}$. The mesostructured $\mathrm{Au} @ \mathrm{TiO}_{2}$ and micropatterned $\mathrm{Au} @ \mathrm{SiO}_{2}$ substrates were sterilized by immersion in $70 \%$ ethanol for $3 \mathrm{~h}$. PA14 Pseudomonas aeruginosa bacteria were streaked onto LB-agar plates and incubated overnight at $37^{\circ} \mathrm{C}$. Single colonies from the agar plates were used to inoculate $10 \mathrm{ml}$ culture in $\mathrm{LB}$ medium at $37^{\circ} \mathrm{C}$, 210 r.p.m. and grown for $20 \mathrm{~h}$. The bacterial culture $(1 \mathrm{ml})$ was washed three times by centrifugation $(8,000 \mathrm{~g} / 3 \mathrm{~min})$ with $10 \mathrm{ml}$ of $\mathrm{LB}$ for removing extracellular pyocyanin, and the final cell pellet was re-suspended in LB to a $D_{600 \mathrm{~nm}}$ of 3.0. The procedure for rinsing off any pyocyanin carry-over from the bacterial suspension was assessed by SERRS for each substrate. The bacterial suspension depleted of pyocyanin was used to start the cultures on the plasmonic platforms with the indicated cell densities at the following final volumes: $5 \mu \mathrm{l}$ on Au@pNIPAM, $50 \mu \mathrm{l}$ on $\mathrm{Au} @ \mathrm{TiO}_{2}$ and $10 \mu \mathrm{l}$ on $\mathrm{Au} @ \mathrm{SiO}_{2}$. The bacterial cultures were grown in a humidified chamber at $28^{\circ} \mathrm{C}(\mathrm{Au} @ \mathrm{pNIPAM}), 30^{\circ} \mathrm{C}\left(\mathrm{Au} @ \mathrm{TiO}_{2}\right)$ and $37^{\circ} \mathrm{C}$ $\left(\mathrm{Au} @ \mathrm{SiO}_{2}\right)$. For growing P. aeruginosa on the $\mathrm{Au} @ \mathrm{TiO}_{2}$ substrate in a semi-solid environment embedded on agar, a small colony of bacteria was grafted with a sterile tip onto the surface of the plasmonic platform. Subsequently, $50 \mu \mathrm{l}$ of molten LB agar $(0.75 \% \mathrm{w} / \mathrm{v})$ was solidified on top and the bacterial colony was incubated in a humidified chamber for $24 \mathrm{~h}$ at $30^{\circ} \mathrm{C}$.

Viable bacteria were quantified by counting colony-forming units (c.f.u.) as follows: samples were removed from planktonic cultures, serially diluted in $\mathrm{LB}$, spread on LB-agar plates and incubated at $37^{\circ} \mathrm{C}$ overnight to determine c.f.u. values. For determination of viable bacteria growing as biofilms on plasmonic platforms, bacteria were scraped off the substrate in $100 \mu \mathrm{l}$ of sterile PBS using an inoculating loop. The samples were bath-sonicated to disperse bacterial aggregates. Next, the bacterial suspension was serially diluted in LB, spread onto LB-agar plates and incubated at $37^{\circ} \mathrm{C}$ overnight to determine c.f.u. values. Bacterial titres were expressed as c.f.u. $\mathrm{ml}^{-1}$.

Detection of spiked pyocyanin in plasmonic substrates implanted in mice. The Au@pNIPAM substrates were doped with $10^{-5}, 10^{-6}$ or $10^{-7} \mathrm{M}$ concentrations of commercial pyocyanin. The substrates were implanted subcutaneously in cadavers of Swiss mice, provided by J. A. Lamas (University of Vigo, Spain). SERS measurements were performed with a $20 \times$ objective for $10 \mathrm{~s}$ and using a maximum power at the sample of $108.6 \mathrm{~mW}$. Animal handling procedures were approved by the Spanish Research Council and the University of Vigo Committee for Animal Experimentation, and they observed the Spanish and European directives for the protection of experimental animals (RD1201/2005; 86/609/EEC).

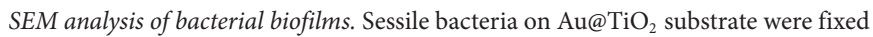
in $2 \%$ glutaraldehyde with $0.1 \mathrm{M}$ cacodylate buffer ( $\mathrm{pH} 7.4)$ for $2 \mathrm{~h}$ at $4{ }^{\circ} \mathrm{C}$. The fixing solution was rinsed with cacodylate buffer $(3 \times 30 \mathrm{~min})$, and samples were dehydrated with a series of increasing concentrations of acetone: $30 \%, 50 \%, 70 \%$, $80 \%$ and $95 \%$ for $30 \mathrm{~min}$, and absolute acetone for $1 \mathrm{~h}$. Next, the sample was subjected to critical point drying with $\mathrm{CO}_{2}\left(73 \mathrm{~atm} ; 31.3^{\circ} \mathrm{C}\right)$ and coated with gold $(10-20 \mathrm{~nm})$. The sample was observed with a JEOL JSM-6700F SEM. Sessile bacteria on $\mathrm{Au} @ \mathrm{SiO}_{2}$ substrate were treated with $4 \%$ formaldehyde at $4{ }^{\circ} \mathrm{C}$ for $12 \mathrm{~h}$. The fixative was washed out with milli-Q water and the sample was observed with a Helios NanoLab 450S SEM.

\section{References}

59. Wang, C., Flynn, N. T. \& Langer, R. Controlled structure and properties of thermoresponsive nanoparticle-hydrogel composites. Adv. Mater. 16, 1074-1079 (2004).

60. Scarabelli, L., Grzelczak, M. \& Liz-Marzan, L. M. Tuning gold nanorod synthesis through prereduction with salicylic acid. Chem. Mater. 25, 4232-4238 (2013).

61. Crepaldi, E. L. et al. Controlled formation of highly organized mesoporous titania thin films: from mesostructured hybrids to mesoporous nanoanatase $\mathrm{TiO}_{2}$. J. Am. Chem. Soc. 125, 9770-9786 (2003).

62. Dong Qin, Y. X. \& Whitesides, G. M. Soft lithography for micro- and nanoscale patterning. Nature Protoc. 5, 491-502 (2010).

63. McDonald, J. C. et al. Fabrication of microfluidic systems in poly(dimethylsiloxane). Electrophoresis 21, 27-40 (2000).

64. Alvarez-Puebla, R. A. et al. Au@pNIPAM colloids as molecular traps for surface-enhanced, spectroscopic, ultra-sensitive analysis. Angew. Chem. Int. Ed. 48, 138-143 (2009).

65. Kern, S. E. \& Newman, D. K. Measurement of phenazines in bacterial cultures. Methods Mol. Biol. 1149, 303-310 (2014) 